2. J. Dieudonne, Recent developments in the theory of locally convex vector spaces, Bull. Amer. Math. Soc. vol. 59 (1953) pp. 495-512.

3. G. W. Mackey, On infinite-dimensional linear spaces, Trans. Amer Math Soc. vol. 57 (1945) pp. 155-207.

4. - On convex topological linear spaces, Trans. Amer. Math. Soc. vol. 60 (1946) pp. 519-537.

NORTHWESTERN UNIVERSITY

\title{
A NOTE ON THE CONTINUITY OF THE INVERSE
}

\section{ROBERT ELLIS}

In his article [2] Wallace mentions the following problem: let $X$ be an algebraic group with a locally compact Hausdorff topology such that the map of $X \times X$ into $X$ which takes $(x, y)$ into $x y$ for all $x, y \in X$ is continuous. Then is $X$ a topological group? The purpose of this note is to answer this question in the affirmative.

Lemma 1 is an immediate consequence of the continuity of multiplication, and the proof of Lemma 2 appears in [1]. The proofs of these lemmas will therefore be omitted.

Lemma 1. Let $F$ be a filter on $X$ such that $F \rightarrow x$ and $F^{-1} \rightarrow y$. Then $y \equiv x^{-1}$.

Lemma 2. Let $A$ be a compact subset of $X$. Then $A^{-1}$ is closed.

Lemma 3. Let $E$ be a countable subset of $X$, and let $x$ be a limit point of $E$. Then $x^{-1}$ is a limit point of $E^{-1}$.

Proof. There is an ultra filter base $\mathcal{U}$ on $E$ such that $\mathfrak{u} \rightarrow x$. By Lemma 1 it is sufficient to show that there is $y \in X$ such that $\mathfrak{u}^{-1} \rightarrow y$. To this end it will be shown that there is a compact set $C$ and a set $U \in \mathcal{U}$ such that $U^{-1} \subset C$.

Let $B=E \cup\{x\}$ and $D=\cup_{n=-\infty}^{\infty} B^{n}$. Then $D$ is a countable subgroup of $X$. Furthermore, if $A=\bar{D}$, then the continuity of multiplication implies that $A^{2} \subset A$.

Now let $V$ be a compact neighborhood of the identity. Then $\bar{D}=A$ implies that $A \subset D V^{-1}$. Thus $A=\mathrm{U}\left[d V^{-1} \cap A \mid d \in D\right]$ $=U\left[d\left(V^{-1} \cap A\right) \mid d \in D\right]$ since $D$ is a group and $A^{2} \subset A$. But $d\left(V^{-1} \cap A\right)$

Received by the editors April 14, 1956. 
is closed for every $d \in D$ by Lemma 2 . Moreover, $A$ is a closed subset of a locally compact space and hence locally compact. This implies that the interior relative to $A$ of one of the sets $d\left(V^{-1} \cap A\right)$ is not null. Hence there is an open set $N$ of $X$ and an element $d$ of $D$ such that $\varnothing \neq N \cap A \subset d\left(V^{-1} \cap A\right)$. Since $\bar{D}=A$, there exists $c \in D \cap N$. Thus $x c^{-1}(N \cap A)=x c^{-1} N \cap A$ is a neighborhood of $x$ relative to $A$. Since $\mathcal{U} \rightarrow x$, and $\mathcal{U}$ is an ultra filter base on $A$, there exists $U \in \mathcal{U}$ such that $U \subset x c^{-1}(N \cap A) \subset x c^{-1} d V^{-1}$. This implies that $U^{-1} \subset V d^{-1} c x^{-1}$ which is compact. The proof is completed.

Lemma 4. Let $A$ be a compact subset of $X$. Then $A^{-1}$ is compact.

Proof. By Lemma $2 A^{-1}$ is closed. The proof will be completed by showing that $A^{-1}$ can be covered by a finite number of translates of an arbitrary compact neighborhood, $V$, of the identity.

Assume this claim false. Then there is a sequence $\left\{x_{n}^{-1}\right\}$ contained in $A^{-1}$ such that $x_{n}^{-1} \notin U\left[x_{i}^{-1} V \mid i=1, \cdots, n-1\right]$. Set $E_{n}=\left[x_{k} \mid k \geqq n\right]$. By the compactness of $A$, there exists $x \in \cap\left[\bar{E}_{n} \mid n=1 \cdots\right]$. Let $U$ be a neighborhood of the identity such that $U^{2} \subset V$. Since $x \in \bar{E}_{1}$, there is $x_{m} \in U x$, whence $x^{-1} \in x_{m}^{-1} U$. Moreover $x \in \bar{E}_{m+1}$ implies by Lemma 3 that $x^{-1} \in E_{m+1}^{-1}$. Thus there is $n>m$ such that $x_{n}^{-1} \in x^{-1} U^{2}$ $\subset x_{m}^{-1} U^{2} \subset x_{m}^{-1} V$, which contradicts the choice of $x_{n}^{-1}$.

THEOREM. Let $X$ be an algebraic group with a locally compact Hausdorff topology such that multiplication is continuous. Then $X$ is a topological group.

Proof. Let $U$ be an open neighborhood of the identity $e$. Let $\mathfrak{e}$ be the collection of compact neighborhoods of $e$. Then it must be shown that there exists $V \in \mathcal{C}$ such that $V^{-1} \subset U$. Suppose this is not the case, i.e. $V^{-1} \cap U^{\prime} \neq \varnothing$ for all $V \in \mathbb{e}$. By Lemma 4 the family $\left(V^{-1} \cap U^{\prime} / V \in \mathbb{C}\right)$ consists of compact sets. Since this family also has the finite intersection property, $\cap\left[V^{-1} \cap U^{\prime} / V \in \mathbb{e}\right] \neq \varnothing$. But $e=\cap\left[V^{-1} / V \in \mathbb{C}\right] \supset \cap\left[V^{-1} \cap U^{\prime} / V \in \mathbb{C}\right]$ implies that $e=\cap\left[V^{-1}\right.$ $\left.\cap \mathcal{u}^{\prime} / V \in \mathbb{e}\right]$. This means in particular that $e \in U^{\prime}$, which is a contradiction. The proof is completed.

\section{REFERENCES}

1. R. Ellis, Continuity and homeomorphism groups, Proc. Amer. Math. Soc. vol. 4 (1953) pp. 969-973.

2. A. D. Wallace, The structure of topological semigroups, Bull. Amer. Math. Soc. vol. 61 (1955) pp. 95-112.

Pennsylvania State University 